\title{
Resting heart rate, functional capacity and prognosis in heart failure patients: atrial fibrillation versus sinus rhythm
}

\begin{abstract}
Background: Higher resting heart rate (HR) is associated with lower exercise capacity and worse prognosis in patients with heart failure (HF). However, recent studies question this relationship in HF patients in atrial fibrillation (AF). We aimed to examine and compare the relationships between resting HR, exercise capacity and outcomes in HF patients in AF and sinus rhythm (SR).

Methods: 282 ambulatory patients with symptomatic HF and left ventricular ejection fraction $\leq 40 \%$ were divided according to rhythm status into SR and AF group. All patients were followed for 60 months and the combined endpoint was defined as cardiac death, urgent heart transplantation or need for mechanical circulatory support.

Results: In the patients enrolled (mean LVEF $27 \pm 7 \%$ ), 19.1\% had AF. The composite endpoint occurred in $24.4 \%$ during follow-up. There were no differences regarding maximal effort, but AF group had lower exercise capacity. In the SR group, there was an inverse relationship between resting HR and exercise capacity ( $\mathrm{r}-0.189, \mathrm{p} 0.004)$. In the $\mathrm{AF}$ group, this relationship was reversed as higher resting HR was associated with better exercise tolerance ( $\mathrm{r} 0.314, \mathrm{p}$ 0.021). Regarding outcomes, patients in SR with a resting HR higher than $72 \mathrm{bpm}$ had higher risk of composite outcome than those with lower resting HR (p 0.033), but this was not evident in AF patients.
\end{abstract}

Conclusion: The impact of resting HR on exercise capacity and prognosis differed entirely between $\mathrm{AF}$ and $\mathrm{SR}$, suggesting that $\mathrm{HR}$ control may need to be managed differently for $\mathrm{AF}$ and $\mathrm{SR}$ in HF patients.

Keywords: heart failure, cardiopulmonary exercise testing, atrial fibrillation
Volume 4 Issue 4 - 2019

\author{
Rita Ilhão Moreira,Tiago Pereira da Silva, \\ António Valentim Gonçalves, Joana Feliciano, \\ Pedro Rio, Rui Soares, Rui Cruz Ferreira \\ Department of Cardiology, Hospital de Santa Marta, Centro \\ Hospitalar de Lisboa Central, Lisbon, Portugal
}

Correspondence: Rita Ilhão Moreira, Department of Cardiology, Hospital de Santa Marta, Centro Hospitalar Lisboa Central. Rua de Santa Marta, n50, I I69-024, Lisbon, Portugal, Tel +351213594000 Email ritailhaomoreira@gmail.com

Received: July 09, 2019 | Published: July 15, 2019
Abbreviations: HF, heart failure; HFrEF, heart failure with reduced ejection fraction; $\mathrm{HR}$, heart rate; $\mathrm{AF}$, atrial fibrillation; $\mathrm{SR}$, sinus rhythm; $\mathrm{pVO}_{2}$, peak oxygen consumption; $\mathrm{VE} / \mathrm{VCO}_{2}$ slope, ventilatory efficiency slope; RER, respiratory exchange ratio

\section{Introduction}

Heart failure (HF) is rapidly growing public health issue that is associated with substantial mortality and morbidity. ${ }^{1}$ Atrial fibrillation (AF) is the most common sustained cardiac arrhythmia in HF, leading to worse prognosis due to an increased thromboembolic risk (mostly stroke) and also, possibly, due to cardiac function impairing. ${ }^{2}$

It is known that medical therapy that reduces resting heart rate (HR) also lowers cardiovascular events in patients with HF, so resting $\mathrm{HR}$ is said to be a modifiable risk factor for $\mathrm{HF}^{3,4}$ In HF patients with sinus rhythm (SR), it has been broadly demonstrated that lower HR confers better exercise capacity and prognosis. ${ }^{5-8}$ Nonetheless, in cases of $\mathrm{AF}$, the clinical repercussion and prognosis significance of resting HR remains unknown.

Thus, this study aimed to examine and compare the relationships between resting HR, functional capacity and outcomes in HF patients in $\mathrm{AF}$ and $\mathrm{SR}$.

\section{Methods}

\section{Selection of patients and evaluation}

Single center analysis with 282 patients with HF with reduced ejection fraction (HFrEF) (left ventricular ejection fraction $\leq 40 \%$ ), that were symptomatic (New York Heart Association class II or III), followed in the Heart Failure Clinics of our center. All patients underwent a comprehensive complementary evaluation, from 2005 to 2014. Clinical, laboratorial, electrocardiographic, echocardiographic, and cardiopulmonary exercise test data were prospectively collected. Patients under 18 years old, comorbidities that limited exercise (including stroke, severe peripheral artery disease or musculoskeletal impairment), planned coronary revascularization, planned cardiac surgery or previous heart transplant were excluded.

A symptom-limited treadmill cardiopulmonary exercise test was performed. The protocol used was the modified Bruce protocol and the treadmill used was GE Marquette Series 2000. Gases (including carbon dioxide production, oxygen uptake and minute ventilation) were analyzed with SensorMedics Vmax 229. The peak oxygen consumption $\left(\mathrm{pVO}_{2}\right)$ was designated as the highest achieved during exercise (30-second average) and was normalized for body mass. Percentage of predicted $\mathrm{pVO}_{2}$ was calculated according to Hansen et al. The ventilatory efficiency $\left(\mathrm{VE} / \mathrm{VCO}_{2}\right)$ slope was determined using data acquired throughout the entire exercise.

\section{Follow-up and endpoint}

Follow-up was performed for 60 months in all patients. Composite endpoint was defined as death, heart transplant not planned (during inotropic therapy or unplanned hospitalization due to HF worsening) or the need for mechanical circulatory support. Data was obtained from the outpatient clinic visits, medical charts review and standardized telephone interview to all patients (12,36 and 60 months). 


\section{Statistical analysis}

Patients were divided into two groups according to baseline rhythm: SR and AF. Data were expressed as percentages and frequencies for categorical variables and as mean \pm standard deviation for continuous variables. Normality was tested using the Shapiro-Wilk or Kolmogorov-Smirnov test as appropriate. Baseline characteristics were compared using the Mann-Whitney or Student's t-test for continuous variables and the Fisher's exact test or Chi-square test for categorical variables. Survival was assessed by Kaplan-Meier analysis and log-rank test. A p-value $<0.05$ was considered significant and the SPSS version 21 software (SPSS Inc., Chicago, Illinois) was used for statistical analysis.

\section{Results}

This study included 282 patients, with mean age of $53.7 \pm 12.1$ years, $75.5 \%$ were male, $19.1 \%$ had AF and $37.6 \%$ had ischemic cardiomyopathy. Left ventricular ejection (LVEF) was $27.4 \pm 7.3 \%$ and $23.0 \%$ were very symptomatic (NYHA class $\geq$ II). Regarding therapy, $96.8 \%$ were taking an angiotensin-converting enzyme inhibitor (ACEi) or angiotensin receptor blocker (ARB), $80.1 \%$ were on a beta-blocker, $68.1 \%$ on a mineralocorticoid antagonist and $26.2 \%$ had biventricular pacing. All patients were followed-up during 60 months and combined endpoint (which includes cardiac death, unplanned heart transplant or need for mechanical circulatory support) occurred in $24.4 \%$ of patients.

\section{Rhythm status}

Patients' characteristics according to rhythm status are shown in Table 1. AF patients more predominantly men, were older, had higher BNP levels and lower Heart Failure Survival Score as compared with SR patients. There were no differences regarding maximal effort (respiratory exchange ratio), but $\mathrm{AF}$ group had lower $\mathrm{pVO} 2$ and higher $\mathrm{VE} / \mathrm{VCO}_{2}$ slope.

Table I Baseline characteristics according to rhythm status

\begin{tabular}{|c|c|c|c|}
\hline & Sinus rhythm $(n=228)$ & Atrial fibrillation $(n=54)$ & $\mathbf{p}$ \\
\hline Age & $52.6 \pm 12.5$ & $58.2 \pm 9.0$ & 0.002 \\
\hline Male gender & $72.80 \%$ & $87.00 \%$ & 0.029 \\
\hline Ischemic aetiology & $40.40 \%$ & $25.90 \%$ & 0.049 \\
\hline NYHA class III (vs. II) & $14.30 \%$ & $9.00 \%$ & $<0.001$ \\
\hline LVEF & $28.4 \pm 2.7 \%$ & $28.5 \pm 0.7 \%$ & 0.116 \\
\hline BNP & $296 \pm 251$ & $489 \pm 191$ & 0.027 \\
\hline $\mathrm{Hb}$ & $13.2 \pm 1.6$ & $12.4 \pm 0.7$ & 0.717 \\
\hline Glomerular filtration rate & $71.6 \pm 11.9$ & $67.8 \pm 31.3$ & 0.006 \\
\hline $\mathrm{Na}$ & $137.6 \pm 1.8$ & $135.0 \pm 2.8$ & 0.156 \\
\hline Resting HR & $82 \pm 15$ & $85 \pm 20$ & 0.739 \\
\hline $\mathrm{PVO}_{2}$ & $18.6 \pm 2.0$ & $13.0 \pm 0.4$ & 0.001 \\
\hline$\% \mathrm{ppVO}_{2}$ & $62.4 \pm 5.8 \%$ & $49.0 \pm 2.8 \%$ & 0.065 \\
\hline $\mathrm{VE} / \mathrm{VCO}_{2}$ slope & $27.3 \pm 3.6$ & $39.3 \pm 7.5$ & 0.002 \\
\hline RER peak & $1.02 \pm 0.02$ & $1.07 \pm 0.08$ & 0.365 \\
\hline HFSS & $8.57 \pm 0.56$ & $8.13 \pm 0.28$ & 0.001 \\
\hline
\end{tabular}

Abbreviations NYHA, New york heart association; LVEF, left ventricular ejection fraction; BNP, brain natriuretic peptide; Hb, haemoglobin; Na, sodium; HR, heart rate; $\mathrm{pVO}_{2}$, peak oxygen consumption; \%ppVO , percentage of predicted peak oxygen consumption; $\mathrm{VE} / \mathrm{VCO}$ slope, ventilatory efficiency slope; RER, respiratory exchange ratio; HFSS, heart failure survival score.

\section{Exercise capacity and prognosis}

Percentage of predicted $\mathrm{pVO}_{2}$ was a strong predictor of prognosis in both SR and AF patients (AUC 0.798, p <0.001 and AUC 0.834, $\mathrm{p}<0.001$, respectively).

The relationship among resting HR and percentage of predicted $\mathrm{pVO}_{2}$ was statistically significant in the SR group, showing an inverse association between resting HR and exercise capacity (Figure 1A). In AF patients, there was also a statistically significant relationship among resting $\mathrm{HR}$ and percentage of predicted $\mathrm{pVO}_{2}$; however, this relationship was reversed as higher resting HR was related to better exercise tolerance (Figure 1B).

Regarding outcomes, the composite endpoint occurred in $24.4 \%$ during follow-up. Patients in SR with a resting HR higher than $72 \mathrm{bpm}$ worse prognosis than those with lower resting HR (Figure 2A). In AF patients, resting HR demonstrated an opposite effect for the composite endpoint though it did not achieve statistical significance (Figure 2B). 

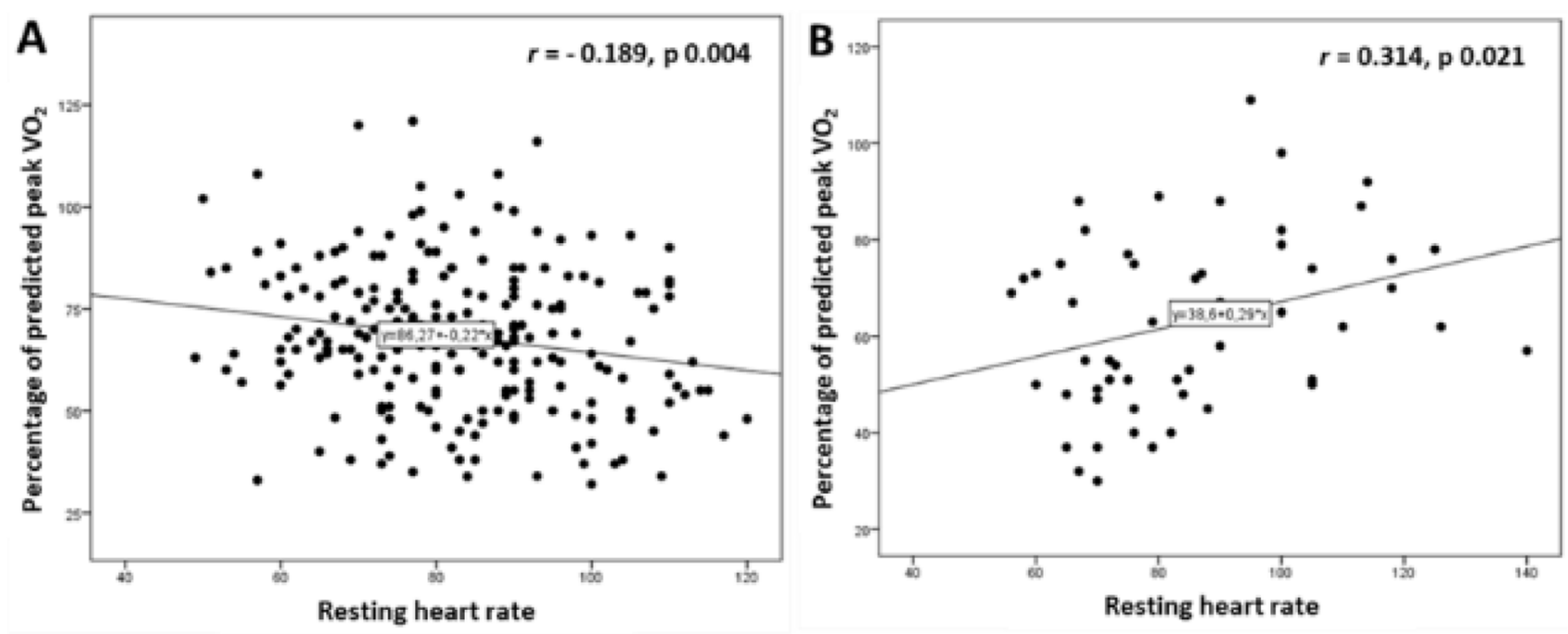

Figure I Relationships between predicted peak oxygen consumption (pVO2) and resting heart rate (HR) in (A) sinus rhythm and (B) atrial fibrillation patients.

A

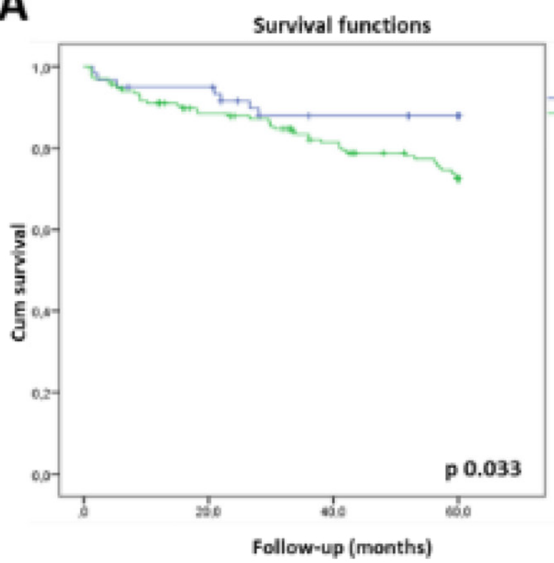

B

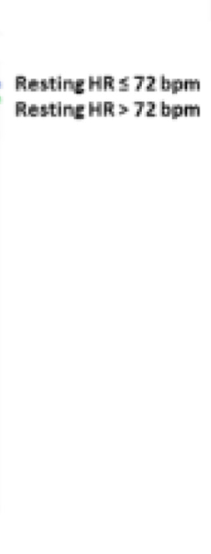

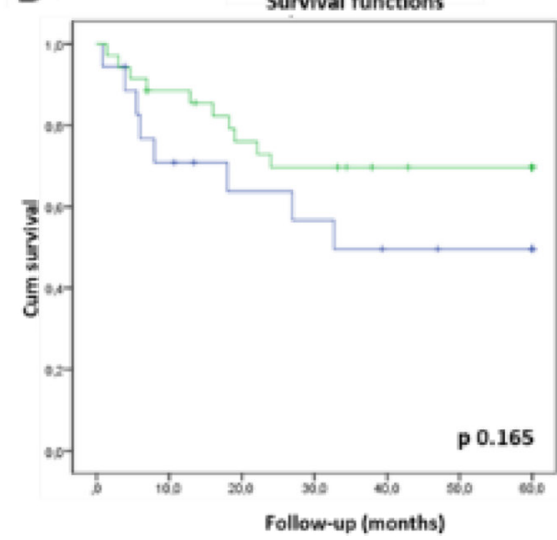

Resting HR $572 \mathrm{bpm}$ Resting $H R>72 \mathrm{bpm}$

Figure 2 Kaplan-Meier analysis according to resting heart rate (HR) in (A) sinus rhythm and (B) atrial fibrillation.

\section{Discussion}

The main findings of this study were that the association among resting $\mathrm{HR}$ and percentage of predicted $\mathrm{pVO}_{2}$ and outcomes contrasted for patients with $\mathrm{AF}$ and $\mathrm{SR}$.

Previous researches have examined the association between resting HR and exercise capacity in the overall population and in heart disease patients, finding an inverse association between resting HR and functional capacity. ${ }^{10-11}$ Moreover, resting HR has been strongly associated with prognosis. $5,6,12$

Chronic HF patients with permanent AF have worse prognosis when compared to those in sinus rhythm. This is due to the fact that new-onset AF in a chronic HF patient impairs cardiac systolic and diastolic function and is also an indicator of a sicker patient. ${ }^{13-15}$

In $\mathrm{HF}$ patients with $\mathrm{AF}$, the optimal resting ventricular rate is still unclear. ${ }^{15-18}$ Few studies have compared resting HR and functinoal capacity in AF patients. Jaber et al. ${ }^{19}$ demonstrate that resting HR was not associated with functional capacity in AF patients with resting heart rat $<90 \mathrm{bpm}$. Kato et al. ${ }^{20}$ showed that the association among resting heart rate and functional capacity was different in SR and AF in the general population. ${ }^{20}$

In our study, we clearly demonstrate that lower resting heart rate is related to better exercise capacity in SR patients yet, in contrary, in $\mathrm{AF}$ patients it is associated with lower exercise capacity. We also demonstrated that resting $\mathrm{HR}<72 \mathrm{bpm}$ is associated with better outcomes in SR but in AF the relationship is inverse.

Therefore, the present study might explain the results of previous studies that showed that ventricular rates lower than $70 \mathrm{bpm}$ are related with unfavourable prognosis in $\mathrm{HF}$ patients with $\mathrm{AF}^{21}$ since $\mathrm{pVO}_{2}$ is well known to be related with future cardiac mortality and morbidity. Our results also help to clarify the causes why beta-blockers titration was not associated with improved prognosis HFrEF patients with $\mathrm{AF}^{22}$ and might also elucidate the relationship between digoxin and adverse events. ${ }^{23-25}$

The pathophysiology of the association between resting heart failure and functional capacity and prognosis in AF patients is still not fully understood. Lewis et al. demonstrated that ventricular rate lowering in AF conferred only a small stroke volume augmentation yet this was counterbalanced rate reduction that conferred lower cardiac. ${ }^{26}$ Additional studies are needed to understand this mechanism.

\section{Limitations}

Generalization of results are limited since this is a single centre analysis. Nevertheless, this allowed the cardio respiratory exercise test protocol being homogeneous in all cases, reducing the interobserver variability. Also, this was a HF population with reduced ejection fraction (mean LVEF systolic $27.4 \pm 7.3 \%$ ) who were able to 
perform exercise and, therefore, the results may not apply to the full HF population. Another limitation is that there was some imbalance between baseline characteristics of SR and AF patients which could be a potential bias, though there were no differences regarding maximal effort during cardiopulmonary exercise test.

\section{Conclusion}

The impact of resting HR on functional capacity and outcomes contrasted entirely between AF and SR in HFrEF patients. This suggests that HR control management may need to be different for AF and SR in HF patients.

\section{Acknowledgments}

This research did not receive any specific grant from funding agencies in the public, commercial, or not-for-profit sectors.

\section{Conflicts of interest}

The authors declare no conflicts of interest.

\section{References}

1. Bleumink GS, Knetsch AM, Sturkenboom MC, et al. Quantifying the heart failure epidemic: prevalence, incidence rate, lifetime risk and prognosis of heart failure The Rotterdam Study. Eur Heart J. 2004;25(18):1614-1619.

2. Kirchhof P, Benussi S, Kotecha D, et al. 2016 ESC Guidelines for the management of atrial fibrillation developed in collaboration with EACTS. Eur J Cardiothorac Surg. 2016;50(5):1-88.

3. Swedberg K, Komajda M, Bohm M, et al. Ivabradine and outcomes in chronic heart failure (shift): A randomised placebo-controlled study. Lancet. 2010;376(9744):875-885.

4. McAlister FA, Wiebe N, Ezekowitz JA, et al. Meta-analysis: Betablocker dose, heart rate reduction, and death in patients with heart failure. Ann Intern Med. 2009;150(11):784-794.

5. Mancini DM, Eisen H, Kussmaul W, et al. Value of peak exercise oxygen consumption for optimal timing of cardiac transplantation in ambulatory patients with heart failure. Circulation. 1991;83(3):778-786.

6. Myers J, Prakash M, Froelicher V, et al. Exercise capacity and mortality among men referred for exercise testing. N Engl J Med. 2002;346:793801 .

7. Kubozono T, Itoh H, Oikawa $\mathrm{K}$, et al. Peak VO2 is more potent than B-type natriuretic peptide as a prognostic parameter in cardiac patients. Circ J. 2008;72(4):575-581.

8. Poole Wilson PA, Uretsky BF, Thygesen K, et al. Mode of death in heart failure: findings from the ATLAS trial. Heart. 2003;89(1):42-48.

9. Guazzi M, Adams V, Conraads V, et al. Clinical recommendations for cardiopulmonary exercise testing data assessment in specific patient populations. Circulation. 2012;126(18):2261-2274.

10. Nauman J, Aspenes ST, Nilsen TI, et al. A prospective population study of resting heart rate and peak oxygen uptake (the HUNT Study, Norway). PLoS One. 2012;7(9):45021.

11. Laukkanen JA, Laaksonen D, Lakka TA, et al. Determinants of cardiorespiratory fitness in men aged 42 to 60 years with and without cardiovascular disease. Am J Cardiol. 2009;103(11):1598-1604.
12. Groote P, Dagorn J, Soudan B, et al. B-type natriuretic peptide and peak exercise oxygen consumption provide independent information for risk stratification in patients with stable congestive heart failure. $J$ Am Coll Cardiol. 2004;43(9):1584-1589.

13. Swedberg K, Olsson LG, Charlesworth A, et al. Prognostic relevance of atrial fibrillation in patients with chronic heart failure on long-term treatment with beta-blockers: results from COMET. Eur Heart J. 2005;26(13):1303-1308.

14. Hoppe UC, Casares JM, Eiskjaer H, et al. Effect of cardiac resynchronization on the incidence of atrial fibrillation in patients with severe heart failure. Circulation. 2006;114(1):18-25.

15. Li SJ, Sartipy U, Lund LH, et al. Prognostic significance of resting heart rate and use of $\beta$-blockers in atrial fibrillation and sinus rhythm in patients with heart failure and reduced ejection fraction: findings from the Swedish Heart Failure Registry. Circ Heart Fail. 2015;8(5):871-879.

16. Hagens VE, Crijns HJGM, Van Veldhuisen DJ, et al. Rate control versus rhythm control for patients with persistent atrial fibrillation with mild to moderate heart failure: results from the RAte Control versus Electrical cardioversion (RACE) study. Am Heart J. 2005;149(6):1106-1111.

17. Van Gelder IC, Hagens VE, Bosker HA, et al. A comparison of rate control and rhythm control in patients with recurrent persistent atrial fibrillation. N Engl J Med. 2002;347(23):1834-1840.

18. Van Gelder IC, Wyse DG, Chandler ML, et al. Does intensity of ratecontrol influence outcome in atrial fibrillation? An analysis of pooled data from the RACE and AFFIRM studies. Europace. 2006;8(11):935-942.

19. Jaber J, Cirenza C, Amaral A, et al. Correlation between heart rate control during exercise and exercise capacity in patients with chronic atrial fibrillation. Clin Cardiol. 2011;34(9):533-536.

20. Kato Y, Suzuki S, Uejima T, et al. The relationship between resting heart rate and peak VO2: a comparison of atrial fibrillation and sinus rhythm. Eur J Prev Cardiol. 2016;23(13):1429-1436.

21. Mareev Y, Cleland JGF. Should b-blockers be used in patients with heart failure and atrial fibrillation? Clin Ther. 2015;37(10):2215-2224.

22. Kotecha D, Holmes J, Krum H, et al. Efficacy of b blockers in patients with heart failure plus atrial fibrillation: an individual-patient data metaanalysis. Lancet. 2014;384(9961):2235-2243.

23. Allen LA, Fonarow GC, Simon DN, et al. Digoxin use and subsequent outcomes among patients in a contemporary atrial fibrillation cohort. $J$ Am Coll Cardiol. 2015;65(25):2691-2698.

24. Gheorghiade M, Fonarow GC, Van Veldhuisen DJ, et al. Lack of evidence of increased mortality among patients with atrial fibrillation taking digoxin: findings from post hoc propensity-matched analysis of the AFFIRM trial. Eur Heart J. 2013;34(20):1489-1497.

25. Turakhia MP, Santangeli P, Winkelmayer WC, et al. Increased mortality associated with digoxin in contemporary patients with atrial fibrillation. J Am Coll Cardiol. 2014;64(7):660-668.

26. Lewis RV, Irvine N, McDevitt DG. Relationships between heart rate, exercise tolerance and cardiac output in atrial fibrillation: the effects of treatment with digoxin, verapamil and diltiazem. Eur Heart J. 1988;9(7):777-781. 